OPEN ACCESS

Edited by:

Antoine Andremont, Paris Diderot University, France

Reviewed by: Bryan Troxell, North Carolina State University, USA Xuesong $\mathrm{He}$ University of California, Los Angeles School of Dentistry, USA

*Correspondence:

Ziad Daoud ziad.daoud@balamand.edu.Ib

Received: 04 October 2016 Accepted: 09 November 2016 Published: 25 November 2016

Citation:

Dahdouh E, Hajjar M, Suarez M and Daoud Z (2016) Acinetobacter baumannii Isolated from Lebanese Patients: Phenotypes and Genotypes of Resistance, Clonality, and Determinants of Pathogenicity. Front. Cell. Infect. Microbiol. 6:163. doi: 10.3389/fcimb.2016.00163

\section{Acinetobacter baumannii Isolated from Lebanese Patients: Phenotypes and Genotypes of Resistance, Clonality, and Determinants of Pathogenicity}

\author{
Elias Dahdouh ${ }^{1}$, Micheline Hajjar ${ }^{2}$, Monica Suarez ${ }^{1}$ and Ziad Daoud ${ }^{2 *}$ \\ ${ }^{1}$ Department of Animal Health, Faculty of Veterinary, Universidad Complutense de Madrid, Madrid, Spain, ${ }^{2}$ Department of \\ Clinical Microbiology, Faculty of Medicine, University of Balamand, Balamand, Lebanon
}

Introduction: Acinetobacter baumannii is a nosocomial pathogen that usually affects critically ill patients. High mortality rates have been associated with MDR A. baumannii infections. Carbapenem resistance among these isolates is increasing worldwide and is associated with certain International Clones (ICs) and oxacillinases (OXAs). Moreover, this organism possesses a wide range of virulence factors, whose expression is not yet fully understood. In this study, clinical $A$. baumannii isolates are characterized in terms of antibiotic resistance, mechanisms of carbapenem resistance, clonality, and virulence.

Materials and Methods: $A$. baumannii clinical isolates $(n=90)$ where obtained from a tertiary care center in Beirut, Lebanon. API 20NE strips in addition to the amplification of blaOXA-51-like were used for identification. Antibiotic susceptibility testing by disk diffusion was then performed in addition to PCRs for the detection of the most commonly disseminated carbapenemases. Clonality was determined by tri-locus PCR typing and doubling times were determined for isolates with varying susceptibility profiles. Biofilm formation, hemolysis, siderophore production, proteolytic activity, and surface motility was then determined for all the isolates. Statistical analysis was then performed for the determination of associations.

Results and Discussion: 81 (90\%) of the isolates were resistant to carbapenems. These high rates are similar to other multi-center studies in the country suggesting the need of intervention on a national level. 74 (91.3\%) of the carbapenem resistant isolates harbored blaOXA-23-like including two that also harbored blaOXA-24-like. 88.9\% of the $A$. baumannii isolates pertained to $\mathrm{ICll}$ and three other international clones were detected, showing the wide dissemination of clones into geographically distinct locations. Virulence profiles were highly diverse and no specific pattern was observed. Nevertheless, an association between motility, siderophore production, and biofilm formation was detected $(p<0.05)$.

Conclusions: A very high rate of carbapenem resistance was detected, showing the need for immediate intervention. IC II and OXA-23-like were the most disseminated, 
reflecting their international dissemination. No specific associations were made between virulence and resistance, but instead associations among certain virulence factors were found. Investigating a more clonally diverse pool of isolates could help in the determination of associations between virulence and resistance.

Keywords: Acinetobacter baumannii, carbapenem resistance, virulence, clonality, oxacillinases

\section{INTRODUCTION}

Acinetobacter baumannii is a nosocomial pathogen that could cause severe secondary infections among critically ill patients (Gordon and Wareham, 2010). This organism has a wide range of intrinsic resistance mechanisms and a heightened ability to acquire resistance to a broad range of antimicrobial agents (Peleg et al., 2012). Mortality rates among critically ill patients infected with Multi-Drug Resistant (MDR) A. baumannii are high, especially when improper empirical treatments are given (Namendys-Silva et al., 2015).

Carbapenems have been the treatment of choice for treating critically ill patients with MDR A. baumannii infections (Breilh et al., 2013). However, the increasing rates of Carbapenem Resistant A. baumannii (CRAB) isolates (Tärnberg et al., 2016) have limited their efficacy and increased mortality rates among infected patients (Lemos et al., 2014). Oxacillinases (OXAs) are the most commonly identified mechanism of carbapenem resistance among A. baumannii isolates. These OXAs include OXA-23-like, OXA-24-like, and OXA-58-like (Nowak and Paluchowska, 2016). A. baumannii also harbors the intrinsic $b l a_{\mathrm{OXA}-51-\text { like }}$ in its genome. This OXA is highly conserved among A. baumannii strains (Turton et al., 2006a), but does not convey resistance to carbapenems unless associated with insertion sequences (Turton et al., 2006b). A few globally disseminated International Clones (ICs) have been found to cause most CRAB infections worldwide and have been associated with the presence of these OXAs (Karah et al., 2012).

A global surveillance program showed that Europe and the Mediterranean regions harbored the highest rate of MDR A. baumannii isolates (Flamm et al., 2016). Moreover, IC II was found to be widely disseminated among these countries (Di Popolo et al., 2011). This clone was also found to be largely disseminated in Lebanon (Rafei et al., 2014). Moreover, CRAB isolates were found to increase in prevalence in this country throughout the past decade (Hamouche and Sarkis, 2012). In 2012, $88 \%$ of 724 A. baumannii isolates recovered from various Lebanese hospitals were found to be resistant to imipenem (Hammoudi et al., 2015a). Although OXA-24-like (Hammoudi et al., 2015b) and OXA-58-like (Zarrilli et al., 2008) have been detected in this country, OXA-23-like seems to be the most prevalent among CRAB isolates (Rafei et al., 2015).

In addition to the various mechanisms of resistance detected among A. baumannii isolates, this organism can deferentially express various virulence factors. These factors include biofilm formation, surface motility, hemolysis on blood agars, siderophore production, and exoprotease activity; among others (Antunes et al., 2011). Whole genome sequencing and insertional mutagenesis led to the identifying of 28 genetic islands coding for genes predicted to be involved in virulence in this organism. Disruption of 6 of these islands did indeed result in avirulent isolates, as shown by infection models using Caenorhabditis elegans and Dictyostelium discoideum (Smith et al., 2007). Among the genes within these islands, genes coding for type IV secretion systems, which are associated with surface motility (Eijkelkamp et al., 2011a), are also found. Of note, A. baumannii was shown to produce different patterns of motility, depending on the choice (Difco Bacto or Eiken) or concentration of the agar (Clemmer et al., 2011). Also among the genes involved in virulence detected in A. baumannii, are genes coding for hemolysins/hemagglutinins (Smith et al., 2007). The expression of these genes was reported on blood agar plates and in liquid assays (Antunes et al., 2011). Exoprotease activity was also reported for A. baumannii strains in the previous study, after prediction of the presence of exoprotease genes in the $A$. baumannii genome (Antunes et al., 2011).

Siderophore production, mainly through the biosynthesis of acinetobactin, was also identified as a virulence determinant in A. baumannii. Successful biosynthesis of this siderophore was shown to be needed for the induction of apoptosis of epithelial cells. Moreover, impairment of siderophore production was shown to reduce the ability of $A$. baumannii to persist in and kill the host, as shown through infection models using Galleria mellonella larvae (Gaddy et al., 2012). Biofilm formation, though at different rates and patterns, has also been reported among A. baumannii clinical isolates (Dahdouh et al., 2016). Several loci in the A. baumannii genome have been implicated with the production of biofilms. One such locus is that coding poly- $\beta$ (1-6)-N-acetylglucosamine, an important component of biofilms, and a virulence factor that is involved in cell to cell adherence and protection from host defenses (Bentancor et al., 2012). Impairment of biofilm production, in addition to pili synthesis and motility, was shown to attenuate virulence in mammalian septicemia models (Cerqueira et al., 2014).

The relationship between virulence and antimicrobial resistance seems to be a highly complex one that is still not completely understood (Peleg et al., 2012). Importantly, the effect of harboring OXAs on virulence in A. baumannii is not well defined (Beceiro et al., 2013). The aim of this study is to characterize A. baumannii isolates obtained from a Lebanese hospital in terms of antibiotic susceptibility, carbapenemases harbored, clonality, and virulence determinants. Moreover, the relationship between virulence and carbapenem resistance will be explored. This would help in providing clinicians and infection control specialists with crucial data that would allow for the development of successful empirical treatments and infection control measures. Moreover, our findings could open the way for the exploitation of the 
interplay between virulence and resistance in the clinical setting.

\section{MATERIALS AND METHODS}

\section{Bacterial Strains}

Ninety five non-repetitive A. baumannii clinical isolates were obtained from Saint George University-University Medical Center (SGH-UMC) over a period extending from June 2013 until August 2014. The isolates were obtained from various patient specimens that include sputum, pus, urine, and blood. The strains were identified using 20NE API strips (BioMérieux, France). The isolates that were not identified as Acinetobacter calcoaceticus-A. baumannii by the biochemical tests were not selected for further investigation. Amplification of the intrinsic $b l a_{\mathrm{OXA}-51-\text { like }}$ gene by PCR was then performed in order to verify that the isolate is A. baumannii (Turton et al., 2006a). The isolates were then stored at $-20^{\circ} \mathrm{C}$ in Luria-Bertani (LB) Broth supplemented with $20 \%$ glycerol until used.

\section{Antimicrobial Susceptibility Testing}

The Kirby Bauer disk diffusion method was performed according to the CLSI guidelines (2014) in order to determine the Antibiotic Susceptibility Profiles (ASTs) of the isolates. The results were interpreted according to the cutoff values of the CLSI document M100-S24 (Clinical and Laboratory Standards Institute, 2014). Antimicrobial agents tested for were cefotaxime $(30 \mu \mathrm{g})$, ceftazidime $(30 \mu \mathrm{g})$, cefepime (30 $\mu \mathrm{g})$, piperacillin/tazobactam $(100 / 10 \mu \mathrm{g})$, meropenem $(10 \mu \mathrm{g})$, imipenem $(10 \mu \mathrm{g})$, trimethoprim/sulfamethoxazole $(1.25 / 23.75$ $\mu \mathrm{g})$, ciprofloxacin $(5 \mu \mathrm{g})$, gentamycin $(10 \mu \mathrm{g})$, and colistin (10 $\mu \mathrm{g})$. In order to verify resistance to colistin, $E$-tests (BioMérieux Mercy l'Etoile, France) and minimum inhibitory concentrations by broth microdilution were performed for isolates that showed narrow inhibition zones around the colistin disks.

\section{Polymerase Chain Reactions}

PCRs for the detection of bla $a_{\text {OXA-51-like }}$ (Turton et al., 2006a);

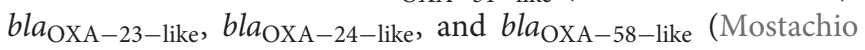
et al., 2009); and bla $a_{\mathrm{OXA}-48}, b l a_{\mathrm{NDM}}$, and bla $a_{\mathrm{KPC}}$ (Poirel et al., 2011) as previously described by the respective authors were performed. International lineage was determined using tri-locus PCR typing as described by Turton et al. (2007). The table summarized by Karah et al. (2012) was used in order to assign the international clonality of the tested isolates. For all PCR reactions, a commercial master mix was used (Qiagen, USA) and the primers, in addition to their respective annealing temperatures, are shown in Table 1. The PCR conditions were an initial elongation at $94^{\circ} \mathrm{C}$ for $3 \mathrm{~min}$; followed by 30 cycles of $94^{\circ} \mathrm{C}$ for $45 \mathrm{~s}$, the respective annealing temperature (Table 1) for $45 \mathrm{~s}$, and $72^{\circ} \mathrm{C}$ for $1 \mathrm{~min}$; and a final extension step at $72^{\circ} \mathrm{C}$ for $5 \mathrm{~min}$. For the $b l a_{\mathrm{OXA}-48}, b l a_{\mathrm{NDM}}$, and $b l a_{\mathrm{KPC}}$ genes, the number of cycles was increased to 36, and the annealing time was increased to $50 \mathrm{~s}$ (Poirel et al., 2011). Positive controls for the respective genes were used from previous studies (Moubareck et al., 2009; Hammoudi et al., 2015b).

\section{Growth Curves}

In order to calculate the doubling time for selected isolates with varying antibiotic susceptibility profiles, 1:100 dilutions from overnight cultures liquid cultures in fresh $\mathrm{LB}$ broth were performed. The fresh suspensions were then incubated at $37^{\circ} \mathrm{C}$ with shaking at $200 \mathrm{rpm}$ for $8 \mathrm{~h}$. Each hour, the $\mathrm{OD}_{600}$ was measured and the doubling times were calculated from the resulting curve as previously described (Hall et al., 2014). The growth curves and all the following experiments were performed in triplicates.

\section{Biofilm Formation}

Biofilm formation was detected in polystyrene tubes after staining with crystal violet as previously described (Tomaras et al., 2003). Briefly, $1 \mathrm{~mL}$ of inoculated LB broth was incubated overnight at $37^{\circ} \mathrm{C}$ and then washed and stained with $1 \%$ crystal violet for 10 min. The dye was then rinsed away and biofilms were visualized and graded as " ++ " if heavy stains were observed and as " + " if faint stains were observed.

\section{Hemolytic Activity}

Hemolytic activity on blood agar plates were tested for by inoculating $10 \mu \mathrm{L}$ of a bacterial suspension adjusted to $10^{6}$ $\mathrm{CFU} / \mathrm{mL}$ in the center of these plates. The plates were then incubated for 6 days at $37^{\circ} \mathrm{C}$ and observed daily (Taybali et al., 2012).

\section{Siderophore Production}

Siderophore production in a liquid medium using the Chrome Azurol Solution (CAS) was performed as previously described (Louden et al., 2011). $5 \mathrm{~mL}$ of the $\mathrm{PMS}_{7}-\mathrm{Ca}$ medium was inoculated and incubated for $72 \mathrm{~h}$. The suspension was then centrifuged at $4000 \times \mathrm{g}$ for $10 \mathrm{~min}$ and $1 \mathrm{~mL}$ of the filter-sterilized supernatant was incubated 1:1 with the CAS. The $\mathrm{OD}_{630}$ was then measured and a $10 \%$ difference between the sample and un-inoculated $\mathrm{PMS}_{7}-\mathrm{Ca}$ with CAS was considered as positive (Machuca and Milagres, 2003).

\section{Surface Motility}

For the detection of surface motility, a single colony was grown in $\mathrm{LB}$ broth overnight at $37^{\circ} \mathrm{C}$. The visual turbidity of the suspension was then adjusted to be equivalent to the $0.5 \mathrm{McF}$ arland standard, in order to contain around $10^{8} \mathrm{CFU} / \mathrm{mL}$. $1 \mu \mathrm{L}\left(10^{5} \mathrm{CFU} / \mathrm{mL}\right)$ of the bacterial suspension was then inoculated on freshly prepared $0.3 \%$ LB-Agar (Difco, BD, USA) plates. The plates were then incubated at $37^{\circ} \mathrm{C}$ for $14 \mathrm{~h}$ and the diameter of the circular diffusion pattern was measured (Clemmer et al., 2011).

\section{Proteolytic Activity}

Proteolytic activity was determined by inoculating $5 \mathrm{~mL}$ of Trypticase Soy Broth Dyalisate with one colony and incubating it overnight at $37^{\circ} \mathrm{C}$ with shaking at $200 \mathrm{rpm}$. The following day, the suspension was centrifuged at $4000 \times \mathrm{g}$ for $10 \mathrm{~min}$ and 500 $\mu \mathrm{L}$ of the filter-sterilized supernatant was incubated with $500 \mu \mathrm{L}$ of $1 \mathrm{mg} / \mathrm{mL}$ Azoalbumin dissolved in $50 \mathrm{mM}$ Tris- $\mathrm{HCl}(\mathrm{pH}=$ 7.7). This preparation was incubated at $37^{\circ} \mathrm{C}$ for $24 \mathrm{~h}$ and $13 \%$ trichloroacetic acid was then added. The tubes were incubated 
TABLE 1 | Primers used in this study with their respective annealing temperatures.

\begin{tabular}{|c|c|c|c|}
\hline Primer & Sequence & Annealing Temperature & References \\
\hline Oxa-51-like-F & 5'-ATGAACATTAAAGCACTC-3' & $46^{\circ} \mathrm{C}$ & Turton et al., 2006b \\
\hline Oxa-51-like-R & 5'-CTATAAAATACCTAATTGTTC-3' & & \\
\hline Oxa-23-like-F & 5'-GATCGGATTGGAGAACCAGA-3' & $53^{\circ} \mathrm{C}$ & Mostachio et al., 2009 \\
\hline Oxa-23-like-R & 5'-ATTTCTGACCGCATTTCCAT-3' & & \\
\hline Oxa-24-like-F & 5'-GGTTAGTTGGCCCCCTTAAA-3' & $53^{\circ} \mathrm{C}$ & Mostachio et al., 2009 \\
\hline Oxa-24-like-R & 5'-AGTTGAGCGAAAAGGGGATT-3' & & \\
\hline Oxa-58-like-F & 5'-AAGTATTGGGGCTTGTGCTG-3' & $53^{\circ} \mathrm{C}$ & Mostachio et al., 2009 \\
\hline Oxa-58-like-R & 5' -CCCCTCTGCGCTCTACATAC-3' & & \\
\hline KPC-F & 5'-CGTCTAGTTCTGCTGTCTTG-3' & $52^{\circ} \mathrm{C}$ & Poirel et al., 2011 \\
\hline KPC-R & 5'-CTTGTCATCCTTGTTAGGCG-3' & & \\
\hline NDM-F & 5'-GGTाTGGCGATCTGGTITC-3’ & $52^{\circ} \mathrm{C}$ & Poirel et al., 2011 \\
\hline NDM-R & 5'-CGGAATGGCTCATCACGATC-3' & & \\
\hline Oxa-48-F & 5'-GCGTGGTTAAGGATGAACAC-3' & $52^{\circ} \mathrm{C}$ & Poirel et al., 2011 \\
\hline Oxa-48-R & 5' -CATCAAGTTCAACCCAACCG-3' & & \\
\hline Group1ompAF306 & 5'-GATGGCGTAAATCGTGGTA-3' & $57^{\circ} \mathrm{C}$ & Turton et al., 2007 \\
\hline Group1and2ompAR660 & 5'-CAACTITAGCGATTTCTGG-3' & & \\
\hline Group1csuEF & 5'-CTITAGCAAACATGACCTACC-3' & $57^{\circ} \mathrm{C}$ & Turton et al., 2007 \\
\hline Group1csuER & 5'-TACACCCGGGTTAATCGT-3' & & \\
\hline Gp1OXA66F89 & 5' -GCGCTTCAAAATCTGATGTA-3' & $57^{\circ} \mathrm{C}$ & Turton et al., 2007 \\
\hline Gp1OXA66R647 & 5' -GCGTATATITTGTTCCATTC-3' & & \\
\hline Group2ompAF378 & 5' -GACCTITCTTATCACAACGA-3' & $57^{\circ} \mathrm{C}$ & Turton et al., 2007 \\
\hline Group1and2ompAR660 & 5'-CAACTITAGCGATTCTGG-3' & & \\
\hline Group2csuEF & 5'-GGCGAACATGACCTATIT-3' & $57^{\circ} \mathrm{C}$ & Turton et al., 2007 \\
\hline Group2csuER & 5'-CTTCATGGCTCGTTGGTT-3' & & \\
\hline Gp2OXA69F169 & 5'-CATCAAGGTCAAACTCAA-3' & $57^{\circ} \mathrm{C}$ & Turton et al., 2007 \\
\hline Gp2OXA69R330 & 5'-TAGCCTIIITTCCCCATC-3' & & \\
\hline
\end{tabular}

at $-20^{\circ} \mathrm{C}$ for $20 \mathrm{~min}$ and centrifuged at $15,000 \times \mathrm{g}$ for $10 \mathrm{~min}$. The $\mathrm{OD}_{440}$ of the supernatant was then measured and $\mathrm{U} / \mathrm{L}$ values were calculated where one $U$ was the amount of enzyme needed to degrade one micromole of Azoalbumin (Ronca-Testoni, 1983; Antunes et al., 2011)

\section{Statistical Analysis}

Normality of the data, when applicable, was tested for using the Shapiro-Wilk test. One-way ANOVA and student $t$-tests were performed for normally distributed data while the KruskalWallis and Mann-Whitney tests were performed non-normally distributed data. Qualitative data was analyzed using the chi squared and the two-sided Fisher's exact tests. $P$-values of less than 0.05 were considered as statistically significant and all tests were performed using the SPSS program, version 17.0 (SPSS 111 Inc., Chicago, USA).

\section{RESULTS}

\section{Bacterial Isolates}

In total, 95 Acinetobacter spp. isolates were collected. Of these isolates, three were Acinetobacter haemolyticus, one was Acinetobacter junii/johnsonii, and one was Acinetobacter radioresistens/lwoffii as identified by the API strips. The rest of the isolates $(n=90)$ were A. baumannii as identified by API and the subsequent amplification of bla $a_{O X A-51-l i k e}$ (Turton et al., 2006a). Thirty five (38.9\%) A. baumannii isolates were collected in 2013 while fifty five (61.1\%) were collected in 2014. Fifty eight (64.5\%) of the A. baumannii isolates were collected from sputum, 17 (18.9\%) from pus, 9 (10\%) from urine, and 4 (4.4\%) from blood samples. Two (2.2\%) isolates were collected from catheters. Thirty six (40\%) of the isolates were obtained from the ICU, 33 $(36.7 \%)$ from general medicine, $10(11.1 \%)$ from the surgical unit, $9(10 \%)$ from the geriatric unit, $1(1.1 \%)$ from the psychiatric unit, and $1(1.1 \%)$ from the pediatric unit.

\section{Antibiotic Susceptibility}

Antibiotic susceptibility profiles were obtained after testing by the Kirby Bauer disk diffusion method according to CLSI guidelines (2014). Eighty one (90\%) of the A. baumannii isolates were resistant to both meropenem and imipenem. Only one isolate was resistant to colistin. Susceptibility to other antimicrobial agents tested for was very low and did not exceed $14.4 \%$ (Table 2). Antibiotic susceptibility profiles are found in Supplementary Table 1.

\section{Dissemination of Carbapenemases and International Clones}

PCRs for the detection of the most common carbapenemases among A. baumannii were performed in addition to tri-locus 
TABLE 2 | Antibiotic susceptibility profiles for 90 A. baumannii isolates collected over a 1-year period.

\begin{tabular}{|c|c|c|c|c|c|c|c|c|c|c|}
\hline \multicolumn{11}{|c|}{ ANTIMICROBIAL AGENT } \\
\hline & \multicolumn{2}{|c|}{ CTX } & \multicolumn{2}{|c|}{ CAZ } & \multicolumn{2}{|c|}{ FEP } & \multicolumn{2}{|c|}{ TZP } & \multicolumn{2}{|c|}{ MEM } \\
\hline & $\mathbf{n}$ & $\%$ & $\mathbf{n}$ & $\%$ & $\mathbf{n}$ & $\%$ & $\mathbf{n}$ & $\%$ & $\mathbf{n}$ & $\%$ \\
\hline Resistant & 79 & 87.8 & 78 & 86.7 & 58 & 64.4 & 81 & 90 & 81 & 90 \\
\hline Intermediate & 9 & 10 & 1 & 1.1 & 19 & 21.1 & 2 & 2.2 & 0 & 0 \\
\hline Sensitive & 2 & 2.2 & 11 & 12.2 & 13 & 14.4 & 7 & 7.8 & 9 & 10 \\
\hline \multicolumn{11}{|c|}{ ANTIMICROBIAL AGENT } \\
\hline & \multicolumn{2}{|c|}{ IMP } & \multicolumn{2}{|c|}{ SXT } & \multicolumn{2}{|c|}{ CIP } & \multicolumn{2}{|c|}{ GT } & \multicolumn{2}{|c|}{ COL } \\
\hline & $\mathbf{n}$ & $\%$ & $\mathbf{n}$ & $\%$ & $\mathbf{n}$ & $\%$ & $\mathbf{n}$ & $\%$ & $\mathbf{n}$ & $\%$ \\
\hline Resistant & 81 & 90 & 83 & 92.2 & 84 & 93.3 & 77 & 85.6 & 1 & 1.1 \\
\hline Intermediate & 0 & 0 & 0 & 0 & 0 & 0 & 0 & 0 & 0 & 0 \\
\hline Sensitive & 9 & 10 & 7 & 7.8 & 6 & 6.7 & 13 & 14.4 & 89 & 98.9 \\
\hline
\end{tabular}

CTX stands for cefotaxime, CAZ for ceftazidime, FEP for cefepime, TZP for piperacillin/tazobactam, MEM for meropenem, IMP for imipenem, SXT for trimethoprim/sulfamethoxazole, CIP for ciprofloxacin, GT for gentamycin, and COL for colistin.

PCR typing which determines international clonality. Of the 81 A. baumannii isolates that showed resistance to carbapenems, $74(91.3 \%)$ harbored $b l a_{\mathrm{OXA}-23-\text { like. Two of these isolates }}$ additionally harbored $b l a_{\text {OXA-24-like. These are isolates } 42 \text { and }}$ 49. None of the other carbapenemases tested for were detected. Seven isolates were resistant to carbapenems but did not harbor any of the tested carbapenemases (Isolates 54, 59, 68, 72, 83, 84, and 85). Two isolates were sensitive to carbapenems but harbored $b l a_{\text {OXA-23-like (Isolates } 17 \text { and } 87) \text {. }}$

Eighty A. baumannii isolates (88.9\%) pertained to IC II, whereas six (6.7\%) pertained to group 4 (Isolates $11,25,39,62$, 82 , and 91 ), one (1.1\%) to group 10 (Isolate 56), and two (2.2\%) to group 14 (Isolates 42 and 72), as summarized by Karah et al. (2012) (Table 3). One A. baumannii isolate showed a pattern that did not pertain to any IC where CsuE and bla OXA-66 were amplified from the first multiplex, and the other allele of CsuE was also amplified from the second multiplex.

\section{Virulence Determinants in Relation with Clonality and Carbapenem Susceptibility}

The result of the various virulence determinants, in addition to international clonality and carbapenem resistance, are shown in Table 3. Seventy seven (85.6\%) of the A. baumannii isolates showed strong biofilm formations while 10 (11.1\%) showed weak formations and $3(3.3 \%)$ showed no biofilm formation. Forty two (46.7\%) of the A. baumannii isolates showed $\alpha$-hemolysis on blood agar plates while one isolate showed $\beta$-hemolysis. Seventy two $(80 \%)$ A. baumannii isolates showed a diffusion pattern indicating surface motility on $0.3 \%$ LB-Agar while 52 (57.8\%) isolates were positive for siderophore production. Proteolytic activity ranged from $4.4 \pm 1.63 \mathrm{U} / \mathrm{L}$ to $61.97 \pm 12.65 \mathrm{U} / \mathrm{L}$ and the doubling times for selected isolates ranged from $0.262 \pm 0.021$ to $0.653 \pm 0.049 \mathrm{~h}$.

No general pattern was observed between the doubling times and antibiotic susceptibility profiles. The slowest doubling time was determined for isolate 28 which was carbapenem-resistant, $\alpha$-hemolytic, produced strong biofilms, showed a motility pattern, harbored bla $a_{\mathrm{OXA}-23-\mathrm{ik} e}$, and pertained to IC II. Nevertheless, other isolates with similar profiles showed faster doubling times (Table 3). One such example is isolate 78 which incidentally had the fastest doubling time. The colistin-resistant isolate 75 showed a similar antibiotic susceptibility pattern and virulence profile to the aforementioned two isolates and had a relatively fast doubling time of $0.328 \pm 0.076 \mathrm{~h}$.

Figure 1 shows representative isolates for non-motile, moderately motile, and highly motile isolates. Isolate 94 showed the highest motility diffusion diameter $(144.5 \pm 7.7 \mathrm{~mm}$ on a $15 \mathrm{~cm}$ square petri dish), produced strong biofilms, showed hemolysis on blood agars, and had a relatively high proteolytic activity $(26.37 \pm 4.42 \mathrm{U} / \mathrm{L})$. This isolate was susceptible to carbapenems and pertained to IC II. Isolate 5 was the only isolate that showed $\beta$-hemolysis and it had the highest proteolytic activity. It also showed a motility diffusion pattern, produced strong biofilms and siderophores, pertained to IC II, and

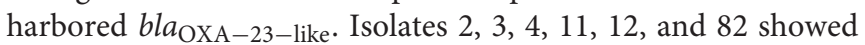
very modest to no motility diffusion patterns and were negative for hemolysis. Isolate 4 also had a slow doubling time (0.594 $\pm 0.036 \mathrm{~h}$ ). However, not all isolates that had similar motility diffusion diameters shared the rest of the virulence profile with these isolates.

Two of the isolates that pertained to group 4 were susceptible to carbapenems and four out of six isolates of this group were negative for siderophore production. Moreover, another two isolates of this group were negative for biofilm formation. Four of the six isolates pertaining to this group harbored $b l a_{\text {OXA-23-like }}$ and were resistant to carbapenems. The isolate pertaining to group ten was susceptible to carbapenems and showed elevated levels of virulence determinants. Both isolates pertaining to Group 14 had similar profiles but one was negative for bla $a_{\text {OXA-23-like }}$ but positive for hemolysis whereas the other harbored $b l a_{\text {OXA-23-like }}$ and $b l a_{\text {OXA-24-like }}$ but was negative for hemolysis. Isolate 18 , which did not pertain to any of the international clones by tri-locus sequence typing was susceptible 
TABLE 3 | Virulence determinants of $90 \mathrm{~A}$. baumannii isolates in addition to international clonality and carbapenem resistance.

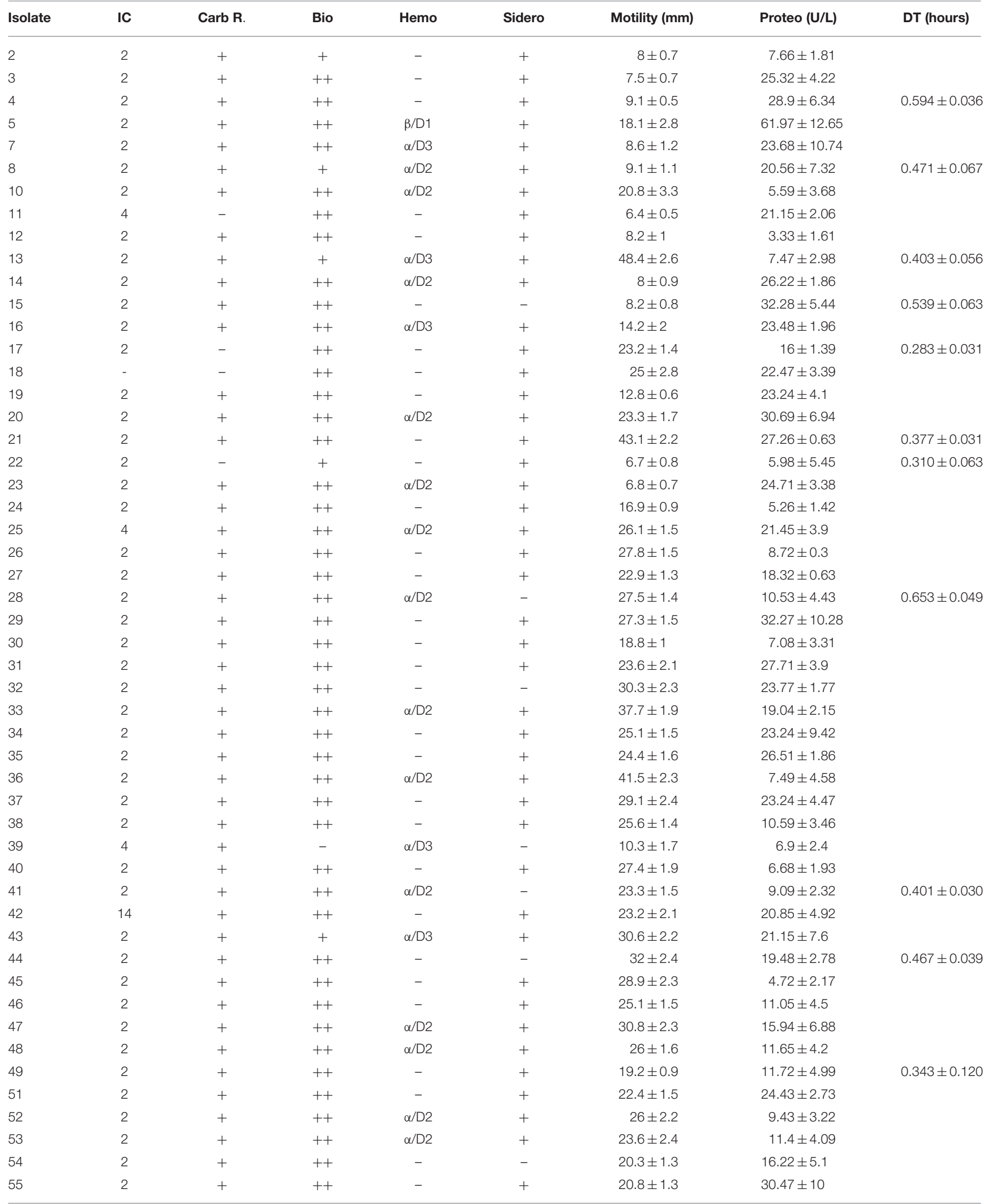

(Continued) 
TABLE 3 | Continued

\begin{tabular}{|c|c|c|c|c|c|c|c|c|}
\hline Isolate & IC & Carb R. & Bio & Hemo & Sidero & Motility (mm) & Proteo (U/L) & DT (hours) \\
\hline 56 & 10 & - & ++ & $\alpha / \mathrm{D} 3$ & + & $31.5 \pm 2.1$ & $11.64 \pm 2.02$ & \\
\hline 57 & 2 & + & + & $\alpha / \mathrm{D} 2$ & - & $21.8 \pm 1.1$ & $25.32 \pm 2.73$ & \\
\hline 58 & 2 & + & - & $\alpha / D 1$ & - & $11.1 \pm 0.6$ & $24.49 \pm 4.55$ & \\
\hline 59 & 2 & + & ++ & $\alpha / \mathrm{D} 2$ & - & $22.1 \pm 1.2$ & $13.81 \pm 4.49$ & \\
\hline 60 & 2 & + & ++ & - & - & $24.6 \pm 1.8$ & $8.37 \pm 2.92$ & \\
\hline 61 & 2 & + & ++ & $\alpha / D 3$ & + & $21.7 \pm 1.1$ & $21.61 \pm 0.24$ & \\
\hline 62 & 4 & - & ++ & $\alpha / D 2$ & - & $23.2 \pm 1.4$ & $7.3 \pm 0.21$ & \\
\hline 63 & 2 & + & ++ & $\alpha / D 1$ & - & $11.1 \pm 0.7$ & $4.4 \pm 1.63$ & \\
\hline 64 & 2 & - & + & $\alpha / D 1$ & - & $13.7 \pm 0.6$ & $10.6 \pm 1.45$ & $0.339 \pm 0.065$ \\
\hline 65 & 2 & + & ++ & $\alpha / D 2$ & - & $25.2 \pm 1.9$ & $7.38 \pm 3.81$ & \\
\hline 66 & 2 & + & ++ & $\alpha / \mathrm{D} 2$ & - & $27.1 \pm 2.2$ & $27.41 \pm 2.87$ & $0.369 \pm 0.021$ \\
\hline 67 & 2 & + & ++ & $\alpha / D 2$ & + & $30.7 \pm 1.9$ & $22.94 \pm 2.87$ & \\
\hline 68 & 2 & + & ++ & - & - & $30 \pm 2.2$ & $22.64 \pm 7.44$ & \\
\hline 69 & 2 & + & ++ & - & - & $31.2 \pm 2.8$ & $28.3 \pm 2.1$ & $0.385 \pm 0.056$ \\
\hline 70 & 2 & + & ++ & $\alpha / \mathrm{D} 2$ & - & $32.3 \pm 1.7$ & $22.05 \pm 6.94$ & \\
\hline 71 & 2 & + & ++ & - & - & $28.3 \pm 2$ & $26.81 \pm 3.1$ & \\
\hline 72 & 14 & + & ++ & $\alpha / D 3$ & + & $20.2 \pm 1.3$ & $22.34 \pm 2.36$ & \\
\hline 73 & 2 & + & ++ & $\alpha / D 1$ & - & $34.7 \pm 2.5$ & $11.08 \pm 5.56$ & \\
\hline 74 & 2 & + & ++ & $\alpha / \mathrm{D} 2$ & - & $28.3 \pm 1.5$ & $9.17 \pm 0.93$ & \\
\hline 75 & 2 & + & ++ & $\alpha / \mathrm{D} 2$ & - & $22.3 \pm 1.8$ & $8.45 \pm 3.1$ & $0.328 \pm 0.076$ \\
\hline 76 & 2 & + & ++ & $\alpha / \mathrm{D} 3$ & + & $24.6 \pm 1.8$ & $27.41 \pm 4.03$ & $0.311 \pm 0.021$ \\
\hline 77 & 2 & + & ++ & - & + & $23.1 \pm 5.9$ & $12.02 \pm 1.96$ & \\
\hline 78 & 2 & + & ++ & - & - & $35.7 \pm 2$ & $12.94 \pm 2.61$ & $0.262 \pm 0.021$ \\
\hline 79 & 2 & + & ++ & - & + & $32.7 \pm 1.8$ & $11.13 \pm 0.69$ & \\
\hline 80 & 2 & + & + & $\alpha / D 2$ & - & $35.5 \pm 1.8$ & $22.94 \pm 2.87$ & \\
\hline 81 & 2 & + & ++ & $\alpha / D 3$ & - & $39.2 \pm 1.5$ & $26.81 \pm 0.89$ & \\
\hline 82 & 4 & + & - & - & - & $11.1 \pm 0.7$ & $8.28 \pm 0.33$ & \\
\hline 83 & 2 & + & + & - & - & $28.6 \pm 2.4$ & $20.85 \pm 4.5$ & \\
\hline 84 & 2 & + & ++ & - & - & $28.9 \pm 2.3$ & $3.21 \pm 3.04$ & \\
\hline 85 & 2 & + & ++ & - & - & $47.8 \pm 2.6$ & $8.49 \pm 2.85$ & \\
\hline 86 & 2 & + & ++ & - & - & $24.6 \pm 1.5$ & $6.54 \pm 4.7$ & \\
\hline 87 & 2 & - & ++ & - & + & $23.4 \pm 1.9$ & $13.9 \pm 4.48$ & \\
\hline 89 & 2 & + & ++ & - & - & $41.5 \pm 2.3$ & $21.81 \pm 8.09$ & \\
\hline 90 & 2 & + & ++ & $\alpha / D 2$ & - & $47.9 \pm 2.9$ & $23.56 \pm 10.31$ & \\
\hline 91 & 4 & + & + & $\alpha / D 3$ & - & $16.2 \pm 1$ & $25.14 \pm 2.31$ & \\
\hline 92 & 2 & + & ++ & - & - & $41.4 \pm 2.1$ & $6.04 \pm 3.49$ & \\
\hline 93 & 2 & + & ++ & $\alpha / \mathrm{D} 3$ & - & $44.3 \pm 2.5$ & $24.55 \pm 9.35$ & \\
\hline 94 & 2 & - & ++ & $\alpha / \mathrm{D} 2$ & - & $144.5 \pm 7.7$ & $26.37 \pm 4.42$ & \\
\hline 95 & 2 & + & ++ & - & - & $46.6 \pm 2.7$ & $23.65 \pm 8.61$ & $0.414 \pm 0.025$ \\
\hline
\end{tabular}

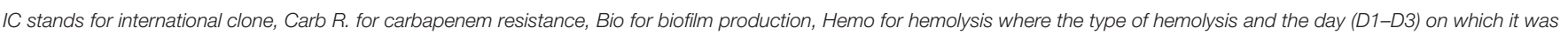
first observed was recorded, Sidero for siderophore production, Proteo for proteolytic activity, and DT for doubling times.

to carbapenems and had similar virulence profiles as isolates pertaining to IC II. Both isolates that harbored bla $a_{\mathrm{OXA}-24-\text { like }}$ in addition to bla $a_{\text {OXA-23-like were negative for hemolysis and had }}$ similar virulence profiles.

\section{Associations between Virulence and Resistance}

IC II was positively associated with both carbapenem resistance and harboring $b l a_{\mathrm{OXA}-23-\mathrm{like}}(p<0.01)$. All the isolates that did not produce biofilms were also negative for siderophore production. The isolates that showed moderate motility diffusion patterns were associated with strong biofilm formation $(p<0.01)$ while those that were either highly motile or non-motile showed a positive association with siderophore production $(p<0.05)$. No other statistical association were made.

\section{DISCUSSION}

In this study, A. baumannii isolates obtained from a major tertiary care center in Beirut, Lebanon were characterized 


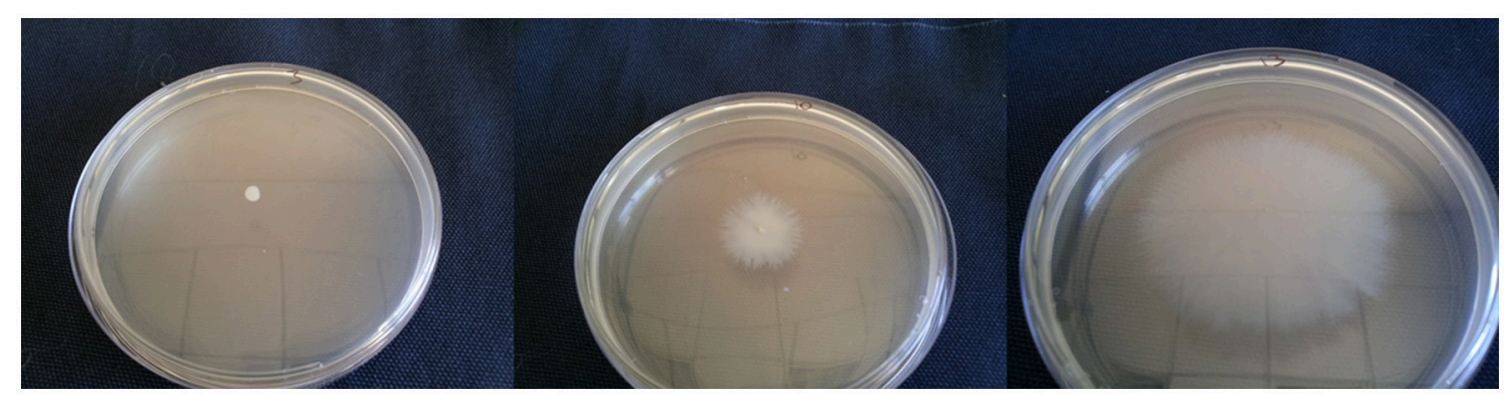

FIGURE 1 | Representative isolates showing (from left to right) no motility, moderate motility, and high motility.

in terms of antibiotic susceptibility, clonality, and virulence determinants. An extremely high rate of carbapenem resistance (90\%) was detected among the A. baumannii isolates. This rate, however, is very similar to that reported from a nation-wide study (Hammoudi et al., 2015a) where the prevalence of CRAB isolates was $88 \%$. These findings suggest an immediate need for the implementation of effective infection control measures and antibiotic stewardship programs in Lebanese hospitals. This need is even more urgent due to the high resistance rates of these isolates to other antimicrobial agents that were tested for in this study (Table 2). The low rate of resistance to colistin among these isolates hold a viable alternative for treatment. Nevertheless, its nephrotoxic effects (Bergen et al., 2012) and the ability of $A$. baumannii to develop resistance toward this antimicrobial agent during therapy (Valencia et al., 2009) limits its effectiveness.

In accordance with other studies performed in Lebanon, IC II was by far the most prevalent clone among the $A$. baumannii isolates (Rafei et al., 2014) and OXA-23-like is the most disseminated (Rafei et al., 2015). These findings are also similar to those reported from other Mediterranean countries (Di Popolo et al., 2011). Interestingly, although an outbreak caused by bla $a_{\mathrm{OXA}-58-\text { like }}$ harboring CRAB isolates was reported from SGH-UMC a few years ago (Zarrilli et al., 2008), this carbapenemase was not detected among our isolates. This could be an indication of the successful eradication of the clone that caused the outbreak at the time of that study.

The presence of $b l a_{\text {OXA-23-like }}$ in two carbapenem-sensitive isolates suggest that either the expression of this gene in these isolates is very modest or that it harbors a mutation that renders it ineffective. Further characterization of these isolates by sequencing the genetic environment of bla $a_{\mathrm{OXA}-23-\text { like }}$ and preforming RT-PCRs could help in better understanding why no carbapenem resistance was detected in these isolates. The seven CRAB isolates in which no carbapenemase was detected could be expressing resistance through carbapenemases that were not tested for in this study and/or through alteration of membrane permeability and efflux pump over-expression (Peleg et al., 2008).

Ten CRAB isolates pertaining to PCR group 4 were first identified in a study investigating A. baumannii isolates from several European countries (Towner et al., 2008). In our study, two out of the six isolates pertaining to this group were susceptible to carbapenems. Similarly, the isolate pertaining to group 10 was sensitive to carbapenems as opposed to the detection of carbapenem resistance among isolates pertaining to this group in a study in Portugal (Grosso et al., 2011). The two isolates pertaining to group 14 were both resistant to carbapenems. One of them did not harbor any of the tested carbapenemases while the other had both OXA-23-like and OXA-24-like. This group was first identified in a study from Romanian hospitals and the isolate pertaining to this group harbored bla $a_{\mathrm{OXA}-58-\text { like }}$ (Bonnin et al., 2011). The diversity of profiles between the isolates pertaining to these groups, in addition to the diversity seen among isolates pertaining to IC II, reflect the plasticity of the A. baumannii genome (Antunes et al., 2014). Moreover, the presence of these clones, in addition to the presence of the globally disseminated IC II (Karah et al., 2012), show the global expansion of A. baumannii clones that are able to expand to wide geographical areas.

A study by Antunes et al. (2011) showed that different $A$. baumannii clinical isolates are able to display different virulence profiles. This was indeed shown to be the case in our study where no specific pattern of virulence was associated with a specific clone (Table 3 ). The A. baumannii isolates investigated had a high degree of variability in terms of virulence profiles. Moreover, no associations between antibiotic susceptibility profiles and doubling times were detected. These findings suggest that each isolate should be treated as a unique case and no general assumptions could be made based on clonality and AST. This also shows that the relationship between virulence and antibiotic resistance is indeed a complex one and warrants further investigation (Peleg et al., 2012). Nevertheless, the low diversity of clones among the tested isolates could be obscuring associations between clonality and virulence, since some isolates pertaining to group 4 and those pertaining to group 14 had similar virulence profiles. Further investigating these associations using larger and more clonally diversified pools could shed further light on the matter. Interestingly, the motility diffusion patterns that were obtained in our study using Difco agar were circular, as opposed to the linear patterns reported while using this kind of agar by Clemmer et al. (2011). The patterns obtained in our study are rather similar to those obtained using Eiken agar. The reason for this difference is still not clear at this point, but could possibly be due to an experimental factor that is independent from the agar brand, or is a property pertaining to the particular strains that 
were tested for in the different studies. This, however, requires further investigation before verification.

Finally, while comparing virulence factors one to another, an association between motility on one hand, and biofilm formation and siderophore production on the other, was determined. The relationship between motility and strong biofilm formation has been previously reported among MDR A. baumannii isolates (Eijkelkamp et al., 2011b). Moreover, the association detected between motility and siderophore production is not surprising since the former factor was associated with biofilm production while the latter allows for iron acquisition that is crucial for biofilm formation (Gentile et al., 2014). These associations reveal a highly complex interplay between the different virulence determinants in $A$. baumannii, especially those that are multi-factorial.

\section{CONCLUSIONS}

In conclusion, a very high rate of carbapenem resistance was detected among clinical A. baumannii isolates obtained from a Lebanese tertiary care center. IC II was the most prevalent clone and OXA-23-like was the most prevalent carbapenemase. The isolates showed highly varied virulence profiles that were not associated with any specific clone or oxacillinase gene. However, associations between motility, biofilm formation, and siderophore production have been found. Increasing the diversity of the pool of isolates could reveal associations between clonality

\section{REFERENCES}

Antunes, L. C., Imperi, F., Carattoli, A., and Visca, P. (2011). Deciphering the multifactorial nature of Acinetobacter baumannii pathogenicity. PLoS ONE 6:e22674. doi: 10.1371/journal.pone.0022674

Antunes, L. C., Visca, P., and Towner, K. J. (2014). Acinetobacter baumannii: evolution of a global pathogen. Pathog. Dis. 71, 292-230. doi: 10.1111/2049632X.12125

Beceiro, A., Tomás, M., and Bou, G. (2013). Antimicrobial resistance and virulence: a successful or deleterious association in the bacterial world? Clin. Microbiol. Rev. 26, 185-230. doi: 10.1128/CMR.00059-12

Bentancor, L. V., O’Malley, J. M., Bozkurt-Guzel, C., Pier, G. B., and MairaLitrán, T. (2012). Poly-N-acetyl- $\beta$-(1-6)-glucosamine is a target for protective immunity against Acinetobacter baumannii infections. Infect. Immun. 80, 651-656. doi: 10.1128/IAI.05653-11

Bergen, P. J., Landersdorfer, C. B., Zhang, J., Zhao, M., Lee, H. J., Nation, R. L., and Li, J. (2012). Pharmacokinetics and pharmacodynamics of 'old' polymyxins: what is new? Diagn. Microbiol. Infect. Dis.. 74, 213-223. doi: 10. 1016/j.diagmicrobio.2012.07.010

Bonnin, R. A., Poirel, L., Licker, M., and Nordmann, P. (2011). Genetic diversity of carbapenem-hydrolysing $\beta$-lactamases in Acinetobacter baumannii from Romanian hospitals. Clin. Microbiol. Infect. 17, 1524-1528. doi: 10.1111/j.14690691.2011.03622.x

Breilh, D., Texier-Maugein, J., Allaouchiche, B., Saux, M. C., and Boselli, E. (2013). Carbapenems. J. Chemother. 25, 1-17. doi: 10.1179/1973947812Y.00000 00032

Cerqueira, G. M., Kostoulias, X., Khoo, C., Aibinu, I., Qu, Y., Traven, A., et al. (2014). A global virulence regulator in Acinetobacter baumannii and its control of the phenylacetic acid catabolic pathway. J. Infect. Dis. 210, 46-55. doi: 10. 1093/infdis/jiu024 and virulence that could allow for the prediction of pathogenicity of a clinical A. baumannii isolate.

\section{AUTHOR CONTRIBUTIONS}

ED: performed the virulence experiments, clonality analysis, part of the PCRs, and statistical analysis. Was also involved in experiment design and data analysis, and drafted the manuscript. MH: performed antibiotic susceptibility analysis and part of the PCRs, obtained the isolates that were included in this work, and was involve in the manuscript preparation. MS: was involved in study design, data analysis, and revision of the manuscript. ZD: was involved in study design, data analysis, revision of the manuscript, and supervised all the work that was done at his laboratory. All authors have approved the final version of the manuscript and are accountable for its content.

\section{ACKNOWLEDGMENTS}

Part of this work has been presented as a poster in the ECCMID conference in April, 2016. The authors would also like to acknowledge CNRS-Lebanon for partly funding the research.

\section{SUPPLEMENTARY MATERIAL}

The Supplementary Material for this article can be found online at: http://journal.frontiersin.org/article/10.3389/fcimb. 2016.00163/full\#supplementary-material
Clemmer, K. M., Bonomo, R. A., and Rather, P. N. (2011). Genetic analysis of surface motility in Acinetobacter baumannii. Microbiology 157, 2534-2544. doi: $10.1099 / \mathrm{mic} .0 .049791-0$

Clinical and Laboratory Standards Institute (2014). Performance Standards for Antimicrobial Susceptibility Testing; Twenty-Fourth Informational Supplement. M100-S24. Wayne, PA: Clinical and Laboratory Standards Institute.

Dahdouh, E., Orgaz, B., Gómez-Gil, R., Mingorance, J., Daoud, Z., Suarez, M., et al. (2016). Patterns of biofilm structure and formation kinetics among Acinetobacter baumannii clinical isolates with different antibiotic resistance profiles. Med. Chem. Commun. 7, 157-163. doi: 10.1039/C5MD00377F

Di Popolo, A., Giannouli, M., Triassi, M., Brisse, S., and Zarrilli, R. (2011). Molecular epidemiological investigation of multidrug-resistant Acinetobacter baumannii strains in four Mediterranean countries with a multilocus sequence typing scheme. Clin. Microbiol. Infect. 17, 197-201. doi: 10.1111/j.1469-0691. 2010.03254.x

Eijkelkamp, B. A., Hassan, K. A., Paulsen, I. T., and Brown, M. H. (2011a). Investigation of the human pathogen Acinetobacter baumannii under iron limiting conditions. BMC Genomics. 12:126. doi: 10.1186/1471-2164-12-126

Eijkelkamp, B. A., Stroeher, U. H., Hassan, K. A., Papadimitrious, M. S., Paulsen, I. T., and Brown, M. H. (2011b). Adherence and motility characteristics of clinical Acinetobacter baumannii isolates. FEMS Microbiol. Lett. 323, 44-51. doi: 10. 1111/j.1574-6968.2011.02362.x

Flamm, R. K., Castanheira, M., Streit, J. M., and Jones, R. N. (2016). Minocycline activity tested against Acinetobacter baumannii complex, Stenotrophomonas maltophilia, and Burkholderia cepacia species complex isolates from a global surveillance program (2013). Diagn. Microbiol. Infect. Dis. 85, 352-355. doi: 10. 1016/j.diagmicrobio.2016.03.019

Gaddy, J. A., Arivett, B. A., McConnell, M. J., López-Rojas, R., Pachón, J., and Actis, L. A. (2012). Role of acinetobactin-mediated iron acquisition functions in the interaction of Acinetobacter baumannii strain ATCC 19606T with human 
lung epithelial cells, Galleria mellonella caterpillars, and mice. Infect. Immun. 80, 1015-1024. doi: 10.1128/IAI.06279-11

Gentile, V., Frangipani, E., Bonchi, C., Minandri, F., Runci, F., and Visca, P. (2014). Iron and Acinetobacter baumannii biofilm formation. Pathogens 3, 704-719. doi: 10.3390/pathogens3030704

Gordon, N. C., and Wareham, D. W. (2010). Multirdug-resistant Acinetobacter baumannii: mechanisms of virulence and resistance. Int. J. Antimicrob. Agents 35, 219-226. doi: 10.1016/j.ijantimicag.2009.10.024

Grosso, F., Quinteira, S., and Peixe, L. (2011). Understanding the dynamics of imipenem-resistant Acinetobacter baumannii lineages within Portugal. Clin. Microbiol. Infect. 17, 1275-1279. doi: 10.1111/j.1469-0691.2011. 03469. $\mathrm{x}$

Hall, B. G., Acar, H., Nandipati, A., and Barlow, M. (2014). Growth rates made easy. Mol. Biol. Evol. 31, 232-238. doi: 10.1093/molbev/mst187

Hammoudi, D., Moubareck, C. A., Hakime, N., Houmani, M., Barakat, A., Najjar, Z., et al. (2015a). Spread of imipenem-resistant Acinetobacter baumannii coexpressing OXA-23 and GES-11 carbapenemases in Lebanon. Int. J. Infect. Dis. 36, 56-61. doi: 10.1016/j.ijid.2015.05.015

Hammoudi, D., Moubareck, C. A., Kanso, A., Nordmann, P., and Sarkis, D. K. (2015b). Surveillance of carbapenem non-susceptible gram negative strains and characterization of carbapenems of classes A, B and D in a Lebanese hospital. Lebanese Med. J. 63, 66-73. doi: 10.12816/0012553

Hamouche, E., and Sarkis, D. K. (2012). [Evolution of susceptibility to antibiotics of Escherichia coli, Klebsiella pneumoniae, Pseudomonas aeruginosa and Acinetobacter baumanii, in a University Hospital Center of Beirut between 2005 and 2009]. Pathol. Biol. 60, e15-e20. doi: 10.1016/j.patbio.2011.03.011

Karah, N., Sundsfjord, A., Towner, K., and Samuelsen, Ø. (2012). Insights into the global molecular epidemiology of carbapenem non-susceptible clones of Acinetobacter baumannii. Drug Resist. Updat. 15, 237-247. doi: 10.1016/j.drup. 2012.06.001

Lemos, E. V., de la Hoz, F. P., Einarson, T. R., McGhan, W. F., Quevedo, E., Castañeda, C., et al. (2014). Carbapenem resistance and mortality in patients with Acinetobacter baumannii infection: systematic review and meta-analysis. Clin. Microbiol. Infect. 20, 416-423. doi: 10.1111/1469-0691. 12363

Louden, B. C., Haarmann, D., and Lynne, A. M. (2011). Use of blue agar CAS assay for siderophore detection. J. Microbiol. Biol. Educ. 12, 51-53. doi: 10.1128/jmbe. v12i1.249

Machuca, A., and Milagres, A. M. (2003). Use of CAS-agar plate modified to study the effect of different variables on the siderophore production by Aspergillus. Lett. Appl. Microbiol. 36, 177-181. doi: 10.1046/j.1472-765X.2003. 01290.x

Mostachio, A. K., van der Heidjen, I., Rossi, F., Levin, A. S., and Costa, S. F. (2009). Multiplex PCR for rapid detection of genes encoding oxacillinases and metallobeta-lactamases in carbapenem-resistant Acinetobacter spp. J. Med. Microbiol. 58, 1522-1524. doi: 10.1099/jmm.0.011080-0

Moubareck, C., Brémont, S., Conroy, M. C., Courvalin, P., and Lambert, T. (2009). GES-11, a novel integron-associated GES variant in Acinetobacter baumannii. Antimicrob. Agents Chemother. 53, 3579-3581. doi: 10.1128/AAC. 00072-09

Ñamendys-Silva, S. A., Correa-García, P., García-Guillén, F. J., González-Herrera, M. O., Pérez-Alonso, A., Texcocano-Becerra, J., et al. (2015). Outcomes of critically ill cancer patients with Acinetobacter baumannii infection. World J. Crit. Care Med. 4, 258-264. doi: 10.5492/wjccm.v4.i3.258

Nowak, P., and Paluchowska, P. (2016). Acinetobacter baumannii: biology and drug resistance - role of carbapenemases. Folia Histochem. Cytobiol. 54, 61-74. doi: 10.5603/FHC.a2016.0009

Peleg, A. Y., de Breij, A., Adams, M. D., Cerqueira, G. M., Mocali, S., Galardini, M., et al. (2012). The success of acinetobacter species; genetic, metabolic and virulence attributes. PLoS ONE 7:e46984. doi: 10.1371/journal.pone.00 46984

Peleg, A. Y., Seifert, H., and Paterson, D. L. (2008). Acinetobacter baumannii: emergence of a successful pathogen. Clin. Microbiol. Rev. 21, 538-582. doi: 10. 1128/CMR.00058-07
Poirel, L., Walsh, T. R., Cuviller, V., and Nordmann, P. (2011). Multiplex PCR for detection of acquired carbapenemase genes. Diagn. Microbiol. Infect. Dis. 70, 119-123. doi: 10.1016/j.diagmicrobio.2010.12.002

Rafei, R., Dabboussi, F., Hamze, M., Eveillard, M., Lemarié, C., Gaultier, M. P., et al. (2014). Molecular analysis of Acinetobacter baumannii strains isolated in Lebanon using four different typing methods. PLoS ONE 9:e115969. doi: 10. 1371/journal.pone.0115969

Rafei, R., Pailhoriés, H., Hamze, M., Eveillard, M., Mallat, H., Dabboussi, F., et al. (2015). Molecular epidemiology of Acinetobacter baumannii in different hospitals in Tripoli, Lebanon using bla(OXA-51-like) sequence based typing. BMC Microbiol. 15:103. doi: 10.1186/s12866-015-0441-5

Ronca-Testoni, S. (1983). Direct spectrophotometric assay for angiotensinconverting enzyme in serum. Clin. Chem. 29, 1093-1096.

Smith, M. G., Gianoulis, T. A., Pukatzki, S., Mekalanos, J. J., Ornston, L. N., Gerstein, M., et al. (2007). New insights into Acinetobacter baumannii pathogenesis revealed by high-density pyrosequencing and transposon mutagenesis. Genes Dev. 21, 601-614. doi: 10.1101/gad.1510307

Tärnberg, M., Nilsson, L. E., and Dowzicky, M. J. (2016). Antimicrobial activity against a global collection of skin and skin structure pathogens: results from the Tigecycline Evaluation and Surveillance Trial (T.E.S.T.), 2010-2014. Int. J. Infect. Dis. 49, 141-148. doi: 10.1016/j.ijid.2016.06.016

Taybali, A. F., Nguyen, K. C., Shwed, P. S., Crosthwait, J., Coleman, G., and Seligy, V. L. (2012). Comparison of the virulence potential of Acinetobacter strains from clinical and environmental sources. PLoS ONE 7:e37024. doi: 10.1371/ journal.pone. 0037024

Tomaras, A. P., Dorsey, C. W., Edelmann, R. E., and Actis, L. A. (2003). Attachment to and biofilm formation on abiotic surfaces by Acinetobacter baumannii: involvement of a novel chaperone-usher pili assembly system. Microbiology. 149, 3473-3484. doi: 10.1099/mic.0.26541-0

Towner, K. J., Levi, K., Vlassiadi, M., and ARPA Steering Group (2008). Genetic diversity of carbapenem-resistant isolates of Acinetobacter baumannii in Europe. Clin. Microbiol. Infect. 14, 161-167. doi: 10.1111/j.1469-0691.2007. 01911.x

Turton, J. F., Gabriel, S. N., Valderrey, C., Kaufmann, M. E., and Pitt, T. L. (2007). Use of sequence-based typing and multiplex PCR to identify clonal lineages of outbreak strains of Acinetobacter baumannii. Clin. Microbiol. Infect. 13, 807-815. doi: 10.1111/j.1469-0691.2007.01759.x

Turton, J. F., Ward, M. E., Woodford, N., Kaufmann, M. E., Pike, R., Livermore, D. M., et al. (2006b).The role of ISAbal in expression of OXA carbapenemase genes in Acinetobacter baumannii. FEMS Microbiol. Lett. 258, 72-77. doi: 10. 1111/j.1574-6968.2006.00195.x

Turton, J. F., Woodford, N., Glover, J., Yarde, S., Kaufmann, M. E., and Pitt, T. L. (2006a). Identification of Acinetobacter baumannii by detection of the blaOXA51-like carbapenemase gene intrinsic to this species. J. Clin. Microbiol. 44, 2974-2976. doi: 10.1128/JCM.01021-06

Valencia, R., Arroyo, L. A., Conde, M., Aldana, J. M., Torres, M. J., FernándezCuenca, F., et al. (2009). Nosocomial outbreak of infection with pan-drug resistant Acinetobacter baumannii in a tertiary care university hospital. Infect. Control Hosp. Epidemiol. 30, 257-263. doi: 10.1086/595977

Zarrilli, R., Vitale, D., Di Popolo, A., Bagattini, M., Daoud, Z., Khan, A. U., et al. (2008). A plasmid-borne blaOXA-58 gene confers imipenem resistance to Acinetobacter baumannii isolates from a Lebanese hospital. Antimicrob. Agents Chemother. 52, 4115-4120. doi: 10.1128/AAC.00366-08

Conflict of Interest Statement: The authors declare that the research was conducted in the absence of any commercial or financial relationships that could be construed as a potential conflict of interest.

Copyright $(0) 2016$ Dahdouh, Haijar, Suarez and Daoud. This is an open-access article distributed under the terms of the Creative Commons Attribution License (CC BY). The use, distribution or reproduction in other forums is permitted, provided the original author(s) or licensor are credited and that the original publication in this journal is cited, in accordance with accepted academic practice. No use, distribution or reproduction is permitted which does not comply with these terms. 